$4-1-2011$

\title{
Torque Around the Center of Mass: Dynamic Stability During Quadrupedal Arboreal Locomotion in the Siberian Chipmunk (Tamias sibiricus)
}

\author{
Andrew R. Lammers \\ Cleveland State University, A.LAMMERS13@csuohio.edu \\ Ulrich Zurcher \\ Cleveland State University, u.zurcher@csuohio.edu \\ Follow this and additional works at: https://engagedscholarship.csuohio.edu/sciphysics_facpub \\ Part of the Physics Commons \\ How does access to this work benefit you? Let us know!
}

\section{Publisher's Statement}

NOTICE: this is the author's version of a work that was accepted for publication in Zoology.

Changes resulting from the publishing process, such as peer review, editing, corrections, structural formatting, and other quality control mechanisms may not be reflected in this document. Changes may have been made to this work since it was submitted for publication. A definitive version was subsequently published in Zoology, 114, 2, April 2011 DOI\#10.1016/ j.zool.2010.11.004

\section{Repository Citation}

Lammers, Andrew R. and Zurcher, Ulrich, "Torque Around the Center of Mass: Dynamic Stability During Quadrupedal Arboreal Locomotion in the Siberian Chipmunk (Tamias sibiricus)" (2011). Physics Faculty Publications. 281.

https://engagedscholarship.csuohio.edu/sciphysics_facpub/281

This Article is brought to you for free and open access by the Physics Department at EngagedScholarship@CSU. It has been accepted for inclusion in Physics Faculty Publications by an authorized administrator of EngagedScholarship@CSU. For more information, please contact library.es@csuohio.edu. 


\title{
Torque around the center of mass: dynamic stability during quadrupedal arboreal locomotion in the Siberian chipmunk (Tamias sibiricus)
}

\author{
Andrew R. Lammers Ulrich Zurcher
}

\section{Introduction}

Locomotion on tree branches, twigs, and foliage presents many challenges to maintaining stability during locomotion (Cartmill, 1985; Preuschoft, 2002). When an animal travels on a horizontal branch, it can topple because of vertical translation, lateral translation, or rolling (Preuschoft, 2002; Stevens, 2003). At any instant during a stride, the animal may lack static stability. This means that the sum of forces acting on the animal's center of mass will cause it to topple or slide off the branch. For example, when the center of mass lies outside the base of support formed by the limbs contacting the substrate, toppling is inevitable unless there is some other mechanism to prevent it. If two or fewer limbs are in contact with the substrate, which occurs during relatively fast locomotion, then static stability is all but impossible. Therefore, dynamic stability becomes progressively more important as velocity rises.

When an animal travels on any substrate, the reaction forces and torques generated by the body's mass and locomotor system work to prevent the animal from collapsing against the accelera- tion of gravity, to propel and brake the animal toward or against the direction of travel, and avoid toppling in any direction. Recent work on terrestrial quadrupeds (Biewener et al., 2009) and humans (Herr and Popovic, 2008) shows that the linear (or translational) and rotational (or angular) movements and accelerations of the body can be explained largely by the magnitude and direction of the substrate reaction force and the distance of the force vector from the body center of mass. In fact, if one imagines that the mass of the entire body of an animal is concentrated in a single point (the center of mass), then the linear and rotational velocity and acceleration of that point can be explained entirely by the substrate reaction force and the distance (moment arm) between the center of mass and that force vector.

Furthermore, Ting et al. (1994), Herr and Popovic (2008), and Biewener et al. (2009) found that the moments around the center of mass in all three axes (yaw, around the vertical axis; roll, around the craniocaudal or fore-aft axis; and pitch, around the mediolateral axis) sum to zero over the course of a single stride. Therefore, even if a torque becomes relatively large, it will be balanced at some time during the stride by a torque in the opposite direction (Ting et al., 1994). This phenomenon can be easily quantified (once the torques around each axis are calculated) by calculating the negative and positive angular impulse (torque $\times$ time, the area under the 
torque versus time graph) around each axis. Thus, even though at any instant there may be a torque acting to destabilize the animal (statically unstable), it is dynamically stable because over the stride time the angular impulses around each axis sum to zero. Furthermore, if the animal is not moving, then net angular impulses will be zero, but the animal will not be dynamically stable. It is necessary that the instantaneous angular impulses are non-zero because these angular impulses contribute to a gyroscopic effect. Such an effect can be observed when a human crosses a stream by running across a fallen log; the mass of the legs rotating around the hip creates a gyroscopic effect that makes the runner dynamically stable (French, 1971).

In this paper we examine arboreal locomotion in the Siberian chipmunk (Tamias sibiricus) as it travels on cylindrical trackways which are narrower than the body of the animal. The chipmunks are semi-arboreal, which means that they are very proficient at traveling on tree branches, but do not regularly move on narrow arboreal substrates (Youlatos et al., 2008). Typically, the closely related Eastern chipmunk (Tamias striatus) only climbs up trees if there is a considerable amount of seeds or fruit available on the twigs and branches (Elliot, 1978; Piaggio and Spicer, 2001). Kawamichi (1989) confirms that Siberian chipmunks forage most of their food on the ground (85.7\%), and only $14.3 \%$ from trees. Thus, the chipmunks are quite proficient at arboreal locomotion, but probably not as specialized for maintaining balance as their tree squirrel relatives. This semi-specialization should increase the likelihood of near-toppling events, where the animals begin to lose their balance but recover. Also, our own observations confirm that the animals usually bound or half-bound when traveling, and that this gait is common at a wide range of speeds $(1-3 \mathrm{~m} / \mathrm{s})$. When the animals bound, the hindlimbs presumably act as one and the forelimbs act as one, which should reduce or eliminate any need to carefully consider the footfall sequence (e.g., lateral sequence diagonal couplet gaits, etc.; Hildebrand, 1976; Schmitt and Lemelin, 2002).

In this study, we seek to test six hypotheses. (1) All angular impulses within a stride sum to zero. (2) Pitch angular impulse (around the mediolateral axis) will be relatively larger than the other angular impulses because of the galloping and bounding motions of the body and limbs. (3) Roll and yaw angular impulses (around the fore-aft and vertical axes, respectively) will be relatively small because the animals usually do not fall off the branch, and they are not turning. Related to hypothesis three: (4) roll and yaw angular impulses will not be associated with each other (e.g., positive yaw impulses will not be associated with positive roll impulses more than what would be expected by random chance). We expect this lack of association between yaw and rolling angular impulses because they control very different aspects of the animals' locomotion. Rolling impulses influence or control the tendency to fall off the sides of the narrow branch. Yaw impulses control the direction of locomotion (e.g., straight ahead, to the right, to the left). (5) We hypothesize that between forelimbs and hindlimbs there will be no differences in any net rolling or yaw angular impulses (i.e., equal net yaw angular impulse between forelimbs and hindlimbs, etc.). (6) Finally, because substrate reaction force usually peaks during the second half of forelimb contact and during the first half of hindlimb contact (Lammers and Biknevicius, 2004), we predict that the timing of peak torques will follow the same pattern.

\section{Materials and methods}

We obtained three adult Siberian chipmunks (two males and one female) through the pet trade. All procedures and animal care methods were approved by the Cleveland State University

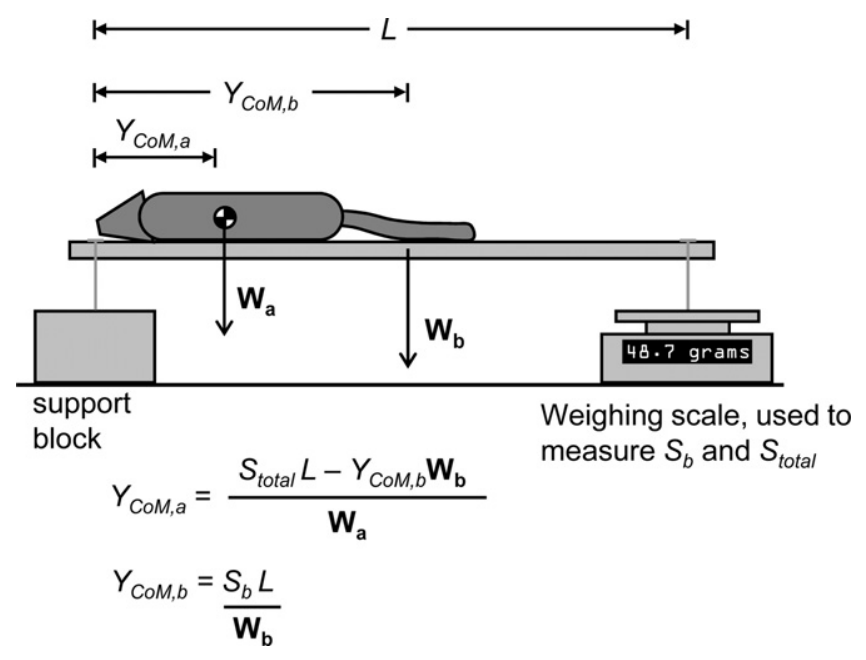

Fig. 1. Balance board method of measuring the location of the center of mass. The weighing scale was used for two measurements: when only the balance board was in place $\left(S_{b}\right)$ and when the balance board and chipmunk were being measured $\left(S_{\text {total }}\right)$ $L=$ the length of the balance board between the two knife points (which were nails); $Y_{C O M, b}=$ the distance between the knife point furthest from the weighing scale and the center of mass of the balance board; $Y_{\mathrm{COM}, a}=$ the distance from the knife point furthest from the weighing scale (and at the tip of the animal's nose) to the animal's center of mass; $W_{a}=$ the weight of the animal; $W_{b}=$ the weight of the balance board.

Institutional Animal Care and Usage Committee and followed $\mathrm{NIH}$ guidelines.

Because the positions and accelerations of the center of mass are considered here, we needed to measure the location of the center of mass in all axes. We used a balance board method, further described by Özkaya and Nordin (1999), Lammers et al. (2006), and Fig. 1. We anaesthetized the chipmunks using $0.3 \mathrm{ml}$ isofluorane so that they could be easily positioned. We placed the individual into a plastic container with a gauze pad, then dropped the isofluorane into the pad and closed the container's lid. The animal typically lost consciousness and became limp within $1 \mathrm{~min}$. The chipmunks typically woke within $1 \mathrm{~min}$, which was usually enough time to pose the animal on the balance board twice. The first pose measured the center of mass location in the fore-aft axis; the chipmunk was placed ventral side down, with the limbs pointing laterally as much as possible. The second pose measured center of mass location in the dorsoventral axis; the animal was placed on its side with the dorsum facing away from the weighing scale. The limbs were posed as much as possible to simulate a standing position. We assumed that the mediolateral position of the center of mass was in the mid-sagittal plane. Because our equipment could resolve the center of mass location within about $0.4 \mathrm{~mm}$, it is unlikely that bilateral asymmetries in mass (e.g., liver on the right, stomach on the left) are detectable using our methods. Mass, body length, and center of mass locations for each animal are indicated in Table 1.

We constructed the arboreal trackway (Fig. 2) from wooden dowel rods covered with 60-grit sandpaper, painted black to increase contrast. The trackway was $178 \mathrm{~cm}$ long and $2 \mathrm{~cm}$ in diameter when covered with the sandpaper. The entire trackway was enclosed by Plexiglas and wood to encourage the chipmunks to remain on the trackway. A removable enclosure at each end provided a shelter for the animals to run into, and also a way to move the chipmunk from the end of the trackway to the beginning between trials. The animals were also trained to run across the trackway, using peanut butter as a reward.

A $3.5 \mathrm{~cm}$ region of the trackway was attached to force transducers so that vertical, fore-aft, and mediolateral components of the substrate reaction force could be measured. We constructed the force pole so that the force-sensitive portion was about $4 \mathrm{~cm}$ 
Table 1

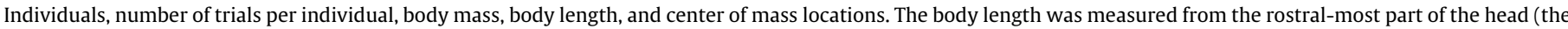

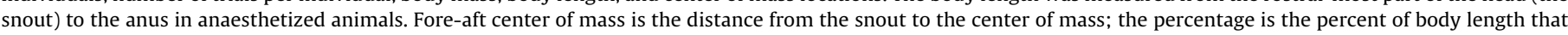

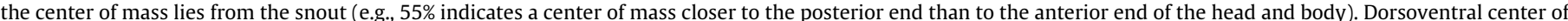

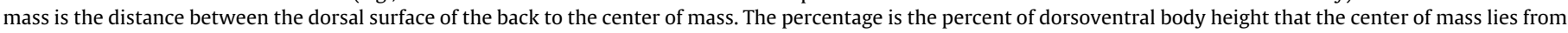
the animal's back. The dorsoventral heights were measured in the thoracic region posterior to the forelimbs; measurements were taken three times and averaged.

\begin{tabular}{|c|c|c|c|c|c|}
\hline Individual & Trials $^{\mathrm{a}}$ & Body mass $(\mathrm{kg})$ & Body length $(\mathrm{cm})$ & Fore-aft center of mass ${ }^{\mathrm{b}}(\mathrm{cm})$ & Dorsoventral center of mass ${ }^{\mathrm{b}}(\mathrm{cm})$ \\
\hline A & $6(3)$ & 0.071 & 14.5 & $7.99(55 \%)$ & $2.12(64 \%)$ \\
\hline $\mathrm{J}$ & $5(4)$ & 0.084 & 15.2 & $8.24(54 \%)$ & $2.04(53 \%)$ \\
\hline $\mathrm{T}$ & $7(4)$ & 0.075 & 14.8 & $7.94(54 \%)$ & $2.11(60 \%)$ \\
\hline
\end{tabular}

a Total number of trials per animal; in parentheses, the number of trials where all hands and feet contacted the force pole.

b These measurements are accurate to within $0.04 \mathrm{~cm}$.

from the transducers; this detail of construction, along with animal training and the Plexiglas and wood enclosure, ensured that the animals stepped on the force pole and not on one of the supporting elements.

Substrate reaction force components were first measured as voltage changes from 6 channels which recorded 2000 samples per second. Signals were passed to a signal conditioning block (SC2345 with SCC-SG04 and SCC-DO01 modules; National Instruments Corp., Austin, TX, USA) and recorded with a virtual instrument program (LabVIEW; National Instruments). Channels 1-4 recorded vertical force, and channels 5 and 6 recorded fore-aft and mediolateral force, respectively. The voltage changes were filtered at $60 \mathrm{~Hz}$ using a moving average technique in Microsoft Excel (average of points $1-33$, then $2-34$, etc.). A second LabVIEW virtual instrument program calibrated voltage changes into force components. Channels 1-4 were summed and then converted via a calibration factor into vertical force.

We used a kinematic method to estimate the coordinates of the center of mass during locomotion. First we anaesthetized each animal before each data collection session, using the same method that we used to measure center of mass location (see above). While the chipmunk was anaesthetized, we marked five intervals $2 \mathrm{~cm}$ apart on the body, starting $2 \mathrm{~cm}$ posterior to the ear, with a blue Sharpie marker (Sanford Corp., Oak Brook, IL, USA) and white nontoxic acrylic paint (Fig. 3). We took advantage of the chipmunk's natural fur coloration by marking the animal on one of the longitudinal light-colored stripes (we also marked intervals on the tail, but we did not use these markings for this experiment because the tail's mass is only about $1 \%$ of the total body mass). The chipmunks were videotaped using two cameras (JVC DVL 9800; JVC, Wayne, NJ, USA) recording at $240 \mathrm{~Hz}$ (Fig. 4). To maintain reasonably high resolution, we had to zoom in and reduce the field of view. As a consequence, we could not capture the entire head, body, and tail throughout an entire stride. The video footage was uploaded to a computer using the ULead VideoStudio 9.0 (ULead Systems, Inc., Taipei, Taiwan). Using the Trimmer module of the APAS motion analysis system (Ariel Dynamics, San Diego, CA, USA), the two video images were synchronized by kinematic event. We also used the Trimmer module to measure forward velocity by recording the times that the animal's nose crossed landmarks painted onto the trackway. Before each set of trials, we calibrated the fields of view by videotaping a stationary, black wooden block with beads glued to it as well as permanent landmarks on the trackway. The fields of view were calibrated such that the long axis of the pole was at zero for the vertical and mediolateral axes; the dimensions of the calibrated field were $14.6 \mathrm{~cm}$ (craniocaudal), $2.0 \mathrm{~cm}$ (vertical), and $3.6 \mathrm{~cm}$ (mediolateral). On the vertical axis, the chipmunks' bodies were partially outside the calibrated volume. To determine if the vertical dimension of the calibrated space resulted in measurement error, we videotaped a block moving through the fields of view. We measured distances between corners of the block with calipers, and then digitized the same corners of the block. We found the difference between measured and digitized distances between corners was less than or equal to $4 \%$. From the videotaped images of the chipmunks we digitized the anterior-most point of the nose (point 1 ), a point on the posterior/ventral part of the ear (point 2), and the five marked points on the body (points 3-7). To estimate the center of pressure in the hand (which has four digits), we digitized

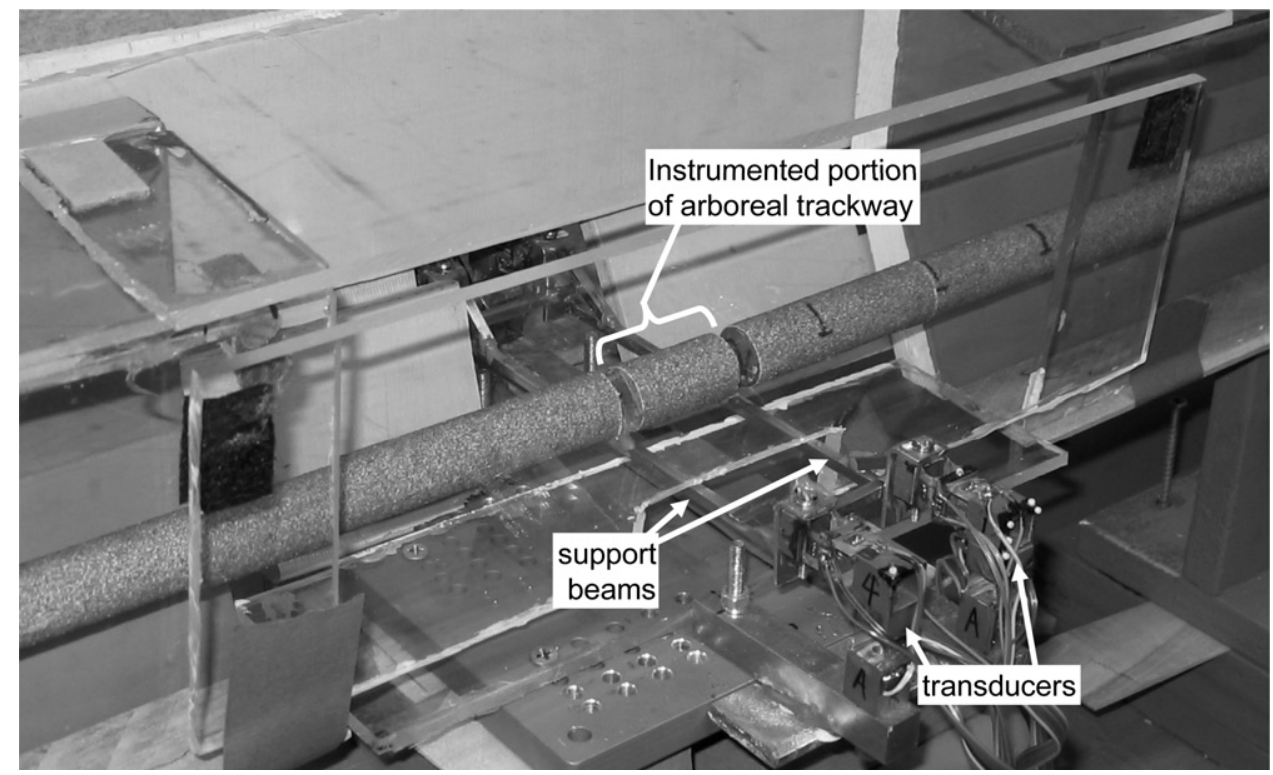

Fig. 2. Arboreal trackway. This photograph was captured before we painted the cylinder black and placed a mirror behind the force pole. 


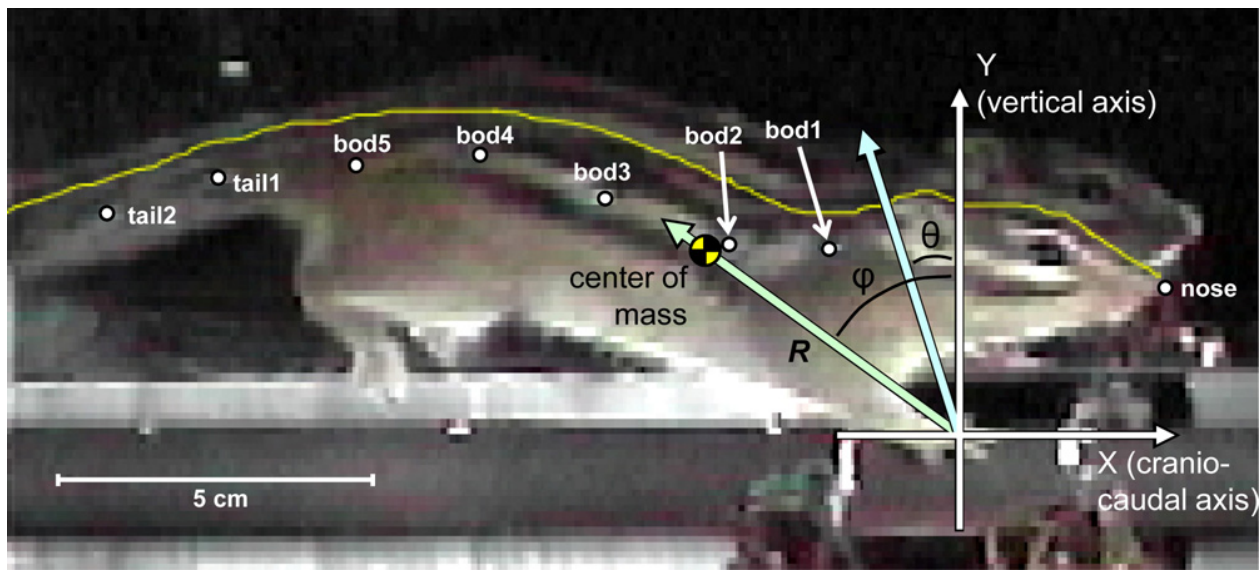

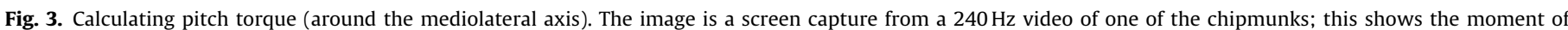

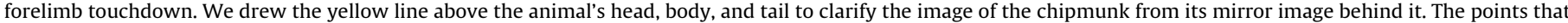

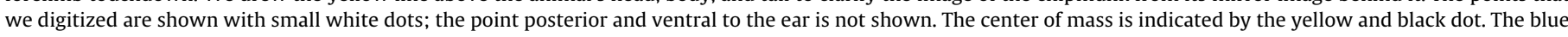

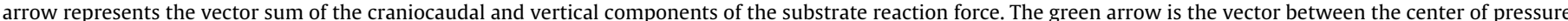

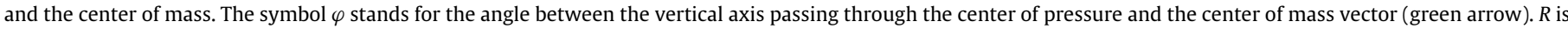
the distance between the center of pressure and center of mass. $\theta$ is the angle between the same vertical axis and the substrate reaction force vector (blue arrow).

two points between the 2nd and 3rd digits; the first was where the digits joined the hand (point 8 ), and the second was between the distal-most points of digits 2 and 3 (point 9). For the foot, which has five digits, we digitized the distal tip and proximal-most parts of digit 3 (points 10 and 11). Using the calibration described above, the 11 coordinates were assembled into three-dimensional Cartesian coordinates using a direct linear transformation in the Transform module of APAS. Using the dorsoventral, mediolateral, and fore-aft distances from each point on the body to the center of mass (measured from the anaesthetized chipmunks), we estimated center of mass location from each individual point (Fig. 5). These coordinates from individual points were averaged to estimate the total body center of mass location; when one or more points were not available because they were out of frame, we used the remaining points to estimate center of mass position. Our method of estimating center of mass location assumes that all segments are of equal mass. During each bounding stride, the body typically undergoes dorsoventral undulation (flexion and extension of the spine), which affects the dorsoventral and fore-aft position of the center of mass

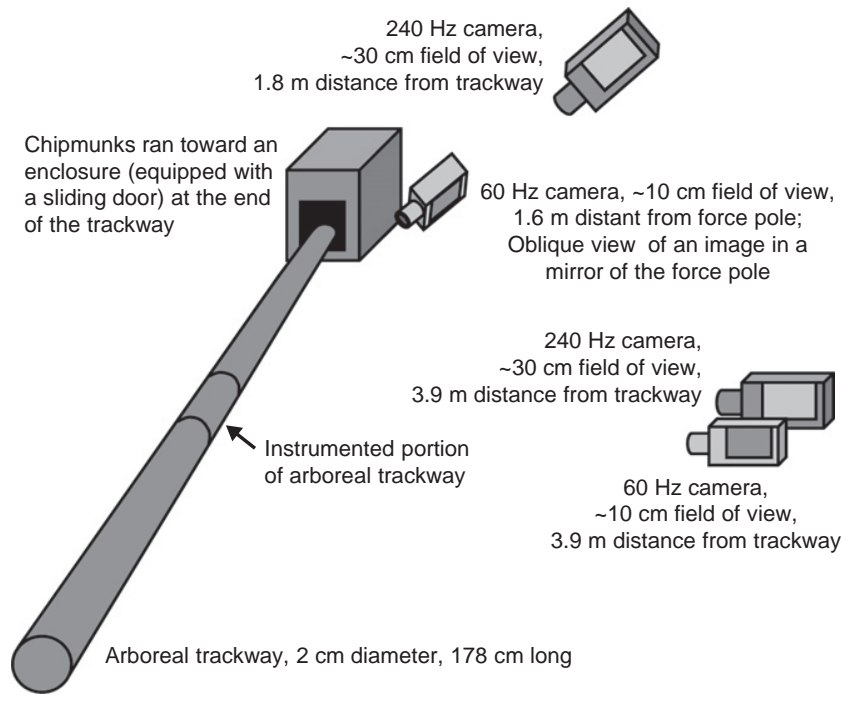

Fig. 4. Schematic of the four video cameras relative to the arboreal trackway. The three cameras on the right side of the figure are in roughly the same plane. Note that the camera distances are not illustrated to scale.
(Nauwelaerts et al., 2009). Our method of estimating center of mass location takes this into account. However, our method is limited in that the limb positions were not taken into account. When we measured center of mass position using the balance board (Fig. 1), we attempted to position the limbs in a standing position, simulating an approximation of the average limb position during locomotion.

As previously indicated, we measured the distances from each chipmunk's vertebral column to the nose, ear, and body coordinates in the mediolateral axis. These measurements were obtained while each chipmunk was under anesthesia. We discovered that the mediolateral distances in the anesthetized animals were quite different from the distances in conscious, running animals. Because

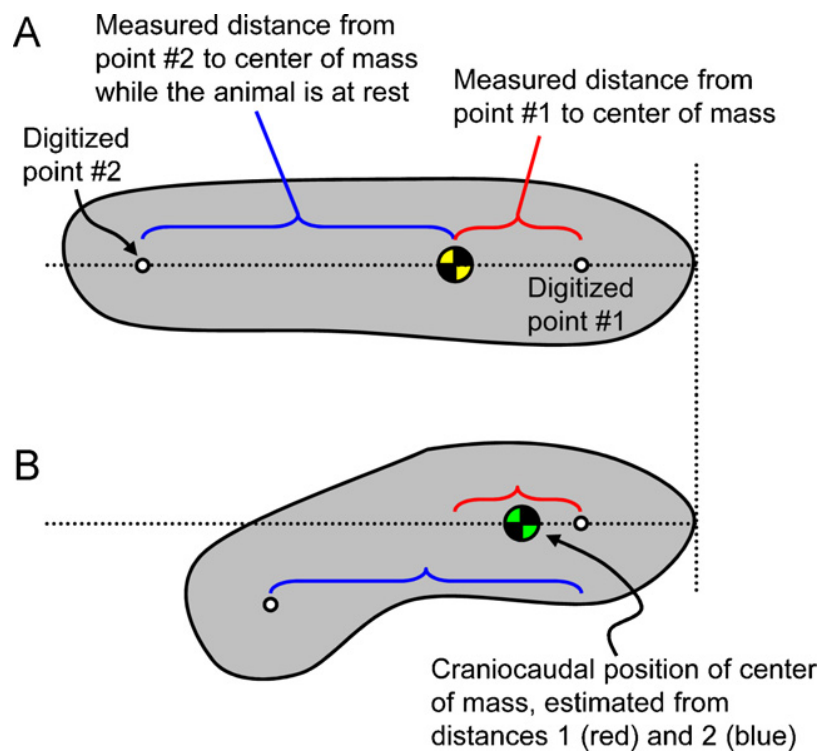

Fig. 5. We measured the craniocaudal and dorsoventral position of the center of mass in each chipmunk while the animal was at rest. In this simplified illustration, only two digitized points (rather than seven) are illustrated. (A) We measured the distance from the actual center of mass (yellow and black point) to the digitized points. Here the animal's body (gray-colored shape) is at rest. (B) The same distances (red and blue brackets) are used to estimate the craniocaudal position of the center of mass. Each bracket yields a different center of mass position because the body has changed shape. We used the average of the distances to estimate the new position of the center of mass (green and black point). For our method we assumed that all body segments were equal in mass. 
the chipmunks always bounded, we assumed that within a stride, the mediolateral position of the center of mass would fluctuate around zero. Thus we calculated the average mediolateral center of mass position within each stride, and then corrected the mediolateral center of mass coordinates using that average such that the coordinates fluctuated around zero.

We needed to use different methods to measure vertical, foreaft, and mediolateral centers of pressure. The vertical coordinate was simply the average of the two hand points or the two foot points. Fore-aft coordinates for hand and foot were calculated the same way, but we also performed a second digitizing session with only one video view to measure the fore-aft distance between right and left limbs, which usually landed within about $1 \mathrm{~cm}$ of each other in the fore-aft axis. For the mediolateral coordinates of the center of pressure, we used a second pair of video cameras (JVC-DF550; JVC, Wayne, NJ, USA), recording at $60 \mathrm{~Hz}$ (Fig. 4). To videotape the right hand and foot, we positioned a camera level with the trackway (in the horizontal plane) $3.9 \mathrm{~m}$ from the trackway. For the left hand and foot, we positioned the second video camera to capture images from a mirror placed on the left side of the trackway. Both cameras captured a very narrow field of view (about $10 \mathrm{~cm}$ ), and both sets of videos captured a lateral view of the force pole. We digitized the same points on the hand and foot as described above, and these four digitized points were also calibrated such that the long axis of the trackway was zero on the vertical axis; the mediolateral axis was orthogonal to the plane of the camera view. We then calculated the position of the mediolateral coordinates by using the vertical coordinates and the dimensions of the branch cylinder; including the approximate thickness of the hand and foot of the chipmunks, we used $1.2 \mathrm{~cm}$ as the radius of the cylinder $(1 \mathrm{~cm}$ force pole radius $+0.2 \mathrm{~cm}$ distal hand or foot thickness $=1.2 \mathrm{~cm}$ ).

We used a LabVIEW virtual instrument to calculate torques around each axis. Fig. 3 illustrates this calculation, using pitch (torque around the mediolateral axis) as an example. First, we calculated the distance between the center of mass and the center of pressure $(R)$ using the coordinates of each. We subtracted center of pressure coordinates from center of mass coordinates so that center of pressure coordinates were all zero. Variables $x_{C o M}$ and $y_{C o M}$ are the fore-aft and vertical coordinates of the center of mass relative to the center of pressure.

$R=\sqrt{x_{C O M}^{2}+y_{C O M}^{2}}$

Next we calculated the angle between the vertical axis and a line drawn between center of mass and center of pressure $(\varphi)$.

$\tan \varphi=\frac{x_{C o M}}{y_{C o M}}$

Then we calculated the magnitude of the force vector $(F)$ formed from vertical and fore-aft components ( $F_{V}$ and $F_{F A}$, respectively).

$F=\sqrt{F_{V}^{2}+F_{F A}^{2}}$

Next we calculated the angle $(\theta)$ between the force vector $F$ and the vertical axis.

$\tan \theta=\frac{F_{F A}}{F_{V}}$

Finally, using the quantities calculated in the four equations above, we calculated the torque around the mediolateral axis ( $\left.\tau_{\text {pitch }}\right)$.

$\tau_{\text {pitch }}=R F \sin (\theta-\varphi)$

We also calculated the torques around the fore-aft axis $\left(\tau_{\text {roll }}\right)$ and vertical axis $\left(\tau_{\text {yaw }}\right)$ in the same way, but using different sets of axes, coordinates of the center of mass, and force components.
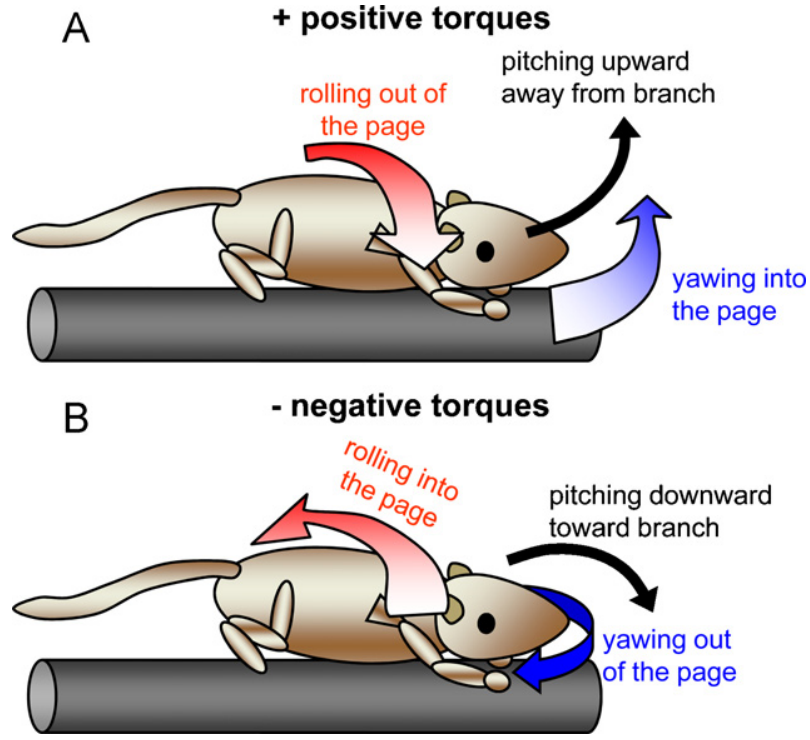

Fig. 6. (A) Positive torques; the animal's body pitches so that the anterior part rises, the body rolls to the right (out of the page), and yaws to the left (into the page). (B) Negative torques; the animal's body pitches so that the anterior part descends closer to the branch, the body rolls to the left (into the page), and yaws to the right (out of the page).

Using the same virtual instrument, we calculated positive and negative angular impulses (pitch, roll, and yaw) from the area contained within the torque versus time plots. We also measured the time of peak negative and positive pitch torque. The direction indicated by positive versus negative torques is illustrated in Fig. 6 .

We divided torque and angular impulse by body weight and average height of the center of mass over the hands and feet coordinates; thus, torque is dimensionless, and angular impulse is dimensionless torque integrated through step duration (s). We then used Systat 11 (Systat Software, Inc., Chicago, IL, USA) for all statistical analyses. We compared forelimbs with hindlimbs for the angular impulses and timing of peak torques using $t$-tests. To determine if positive or negative rolling torques were associated with positive or negative yaw torques, we put each trial into one of four categories (positive yaw, positive roll; positive yaw, negative roll; negative yaw, positive roll; and negative yaw, negative roll). We then used a chi-square test to determine if each yaw direction was associated with a particular roll direction more often than what would be predicted by random assortment. We also calculated Pearson correlation coefficients between yaw and rolling angular impulses separately for the forelimbs and the hindlimbs.

\section{Results}

Torques and angular impulses over the course of the entire stride

These results are based on 11 trials where all four limbs contacted the force pole, forelimbs first, followed by hindlimbs. Pitch angular impulse (moment around the mediolateral axis passing through the center of mass multiplied by total contact time) was the greatest in magnitude; yaw and roll angular impulses were roughly equivalent in magnitude (Table 2 and Fig. 7). Usually all torques for the entire stride began and ended near zero (Fig. 8). In each stride, net angular impulses in all three directions were rarely close to zero. When mean net angular impulses were converted into percentages of the mean positive and mean negative angular impulses, we found that net roll angular impulse was closest to zero, followed by net pitch and then net yaw angular impulses. However, it is apparent 
Table 2

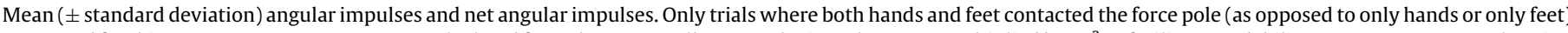

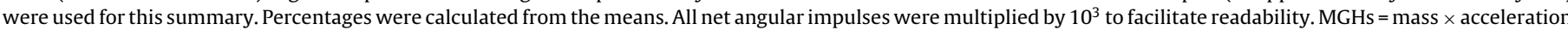
of gravity $\times$ average center of mass height $\times$ time.

\begin{tabular}{|c|c|c|c|c|c|}
\hline & Positive & Negative & Net & Net, \% of positive & Net, \% of negative \\
\hline Pitch $\left(\mathrm{MGHs} \times 10^{3}\right)$ & $35.4 \pm 18.1$ & $-28.2 \pm 17.0$ & $7.1 \pm 24.6$ & 20 & 25 \\
\hline Yaw $\left(\right.$ MGHs $\left.\times 10^{3}\right)$ & $4.7 \pm 4.6$ & $-6.9 \pm 4.6$ & $-2.2 \pm 4.7$ & 47 & 32 \\
\hline Roll (MGHs $\times 10^{3}$ ) & $8.1 \pm 8.2$ & $-6.9 \pm 8.9$ & $1.1 \pm 15.6$ & 14 & 16 \\
\hline
\end{tabular}

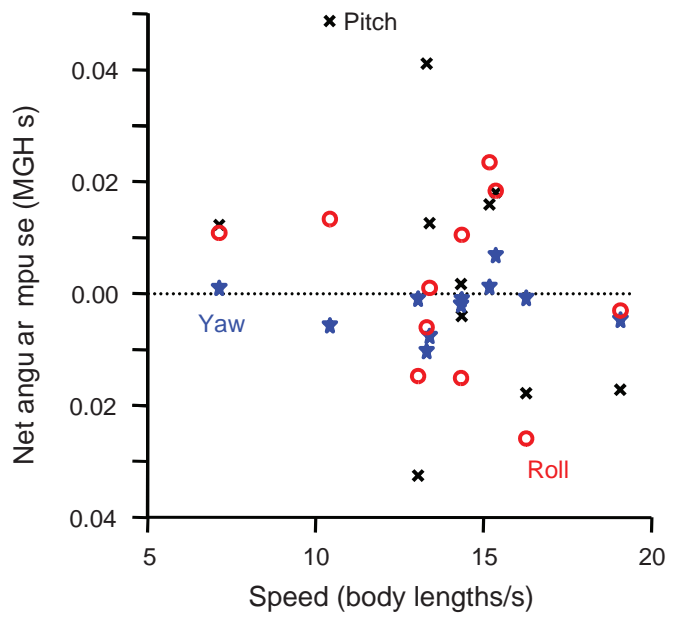

Fig. 7. Net angular impulses versus speed for trials where all four limbs contacted the force pole. The units for angular impulse are [individual mass $\times$ acceleration of gravity $\times$ average center of mass height $\times$ time]; for example, a value of 0.04 indicates that a force equivalent to $4 \%$ of the animal's body weight is applied through a lever arm equivalent in length to the average center of mass height (about 3-5 cm) during one step (about $0.05 \mathrm{~s}$ ). Xs represent pitch, stars are yaw, and circles are rolling angular impulses. Net angular impulse values close to zero indicate that over the course of the step, the positive and negative torques around that particular axis are balanced; see Fig. 4 for illustration of positive and negative torques.

in Fig. 7 that net yaw angular impulses cluster closest to zero, and appear to be the most balanced.

\section{Torques and angular impulses generated by pairs of limbs}

We examined 18 trials, where forelimbs are represented by 14 trials and hindlimbs by 15 trials; in 11 of these cases, both fore- and hindlimbs contacted the force pole. There were no apparent differences among the three individuals, so we disregarded individual as a significant factor for all analyses and comparisons.

After a brief aerial phase, the forelimbs touched down, generating positive pitch torque (Fig. 8A). Near the end of the stance phase of the forelimbs, the pitch torque usually became negative. At about the same time that forelimbs lifted off, the hindlimbs touched down simultaneously. Pitch torque was almost always negative during the stance phase of the hindlimbs (Fig. 8B). The pitch angular impulses (positive, negative, and net) were significantly different between fore- and hindlimbs $(P \leq 0.0009$; Table 3$)$. Yaw and rolling torques had few apparent patterns (Fig. 8C-F). In the forelimbs, the yaw torque usually reached its peak before the first half of the step (Fig. 8C). Also, rolling torques were often higher during the stance phase of the forelimbs as compared with hindlimbs (Fig. 8D), although there were no significant differences in angular impulses between limbs.

Although the chipmunks always used bounding gaits, the right and left limb pairs rarely landed without some fore-aft offset - for example, the right hand might land a centimeter in front or behind the left hand. Furthermore, the mediolateral center of pressure (average of the center of pressure of the right and left hands or feet) was also rarely in the centerline of the branch. Therefore we used least-squares regression to test the influence of fore-aft or mediolateral offset on the net yaw and rolling angular impulses. Using offset (fore-aft or mediolateral) as the independent variable and net torque (yaw or roll) as the dependent one, we found that net roll was significantly correlated with mediolateral offset $(P=0.0002$; Fig. 9). When the center of pressure was on the left side of the branch, most or all of the rolling angular impulse was also directed toward the left side (thus pushing the animal's center of mass back to the right). The reverse was true when the center of pressure was on the right side of the branch. All other correlations were not significant $(P \geq 0.37)$. The chipmunks strongly favored the left side of the branch trackway; the estimated center of pressure was on the left side in 22 of the 29 limb contacts.

The relationship between net rolling torque and net yaw torque is illustrated in Fig. 10. A chi-square test indicates random distribution. Therefore net rolling torques to the left or right were not associated with net yaw torques to the left or right more than what would be expected from random pairing. However, when forelimbs and hindlimbs were considered separately, there appeared to be a positive relationship between forelimb net yaw and net rolling angular impulses. Conversely, there appeared to be a negative relationship between hindlimb net yaw and rolling angular impulses. To test for the possibility of association between yaw and rolling angular impulses, we used calculated Pearson correlation coefficients separately for forelimbs and hindlimbs. The forelimbs' correlation coefficient was 0.854 , and the hindlimbs' coefficient was -0.586 (Fig. 10).

\section{Timing of maximum positive and minimum negative torques}

We converted the timing of peak torques (positive and negative) into percent of step duration (Table 4). Mean peak positive pitch in the forelimbs occurred during the first half of the step $(40 \pm 6 \%)$; the forelimbs' pitch torque became negative late in the step ( $92 \pm 7 \%)$. The timing of peak negative pitch torque was significantly different between fore- and hindlimbs $(P \leq 0.0001)$, but there were no other

Table 3

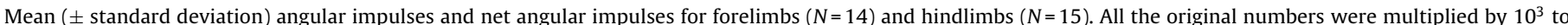
facilitate readability. MGHs = mass $\times$ acceleration of gravity $\times$ average center of mass height $\times$ time.

\begin{tabular}{|c|c|c|c|c|c|c|}
\hline \multirow[t]{2}{*}{ Angular impulses } & \multicolumn{2}{|l|}{ Positive } & \multicolumn{2}{|l|}{ Negative } & \multicolumn{2}{|l|}{ Net } \\
\hline & Forelimbs & Hindlimbs & Forelimbs & Hindlimbs & Forelimbs & Hindlimbs \\
\hline Pitch $\left(\mathrm{MGHs} \times 10^{3}\right)$ & $33.2 \pm 17.9^{*}$ & $1.1 \pm 2.5$ & $-2.1 \pm 2.5^{*}$ & $-21.2 \pm 17.7$ & $31.1 \pm 19.4^{*}$ & $-20.2 \pm 18.9$ \\
\hline Yaw $\left(\right.$ MGHs $\left.\times 10^{3}\right)$ & $1.9 \pm 2.9$ & $2.7 \pm 4.4$ & $-4.0 \pm 4.3$ & $-1.9 \pm 1.5$ & $-2.1 \pm 6.5$ & $0.8 \pm 4.9$ \\
\hline Roll (MGHs $\times 10^{3}$ ) & $5.2 \pm 6.0$ & $3.7 \pm 3.5$ & $-4.1 \pm 6.7$ & $-1.9 \pm 3.0$ & $1.1 \pm 11.2$ & $1.7 \pm 5.9$ \\
\hline
\end{tabular}

\footnotetext{
${ }^{*}$ Forelimbs and hindlimbs significantly different, $P \leq 0.0009$.
} 

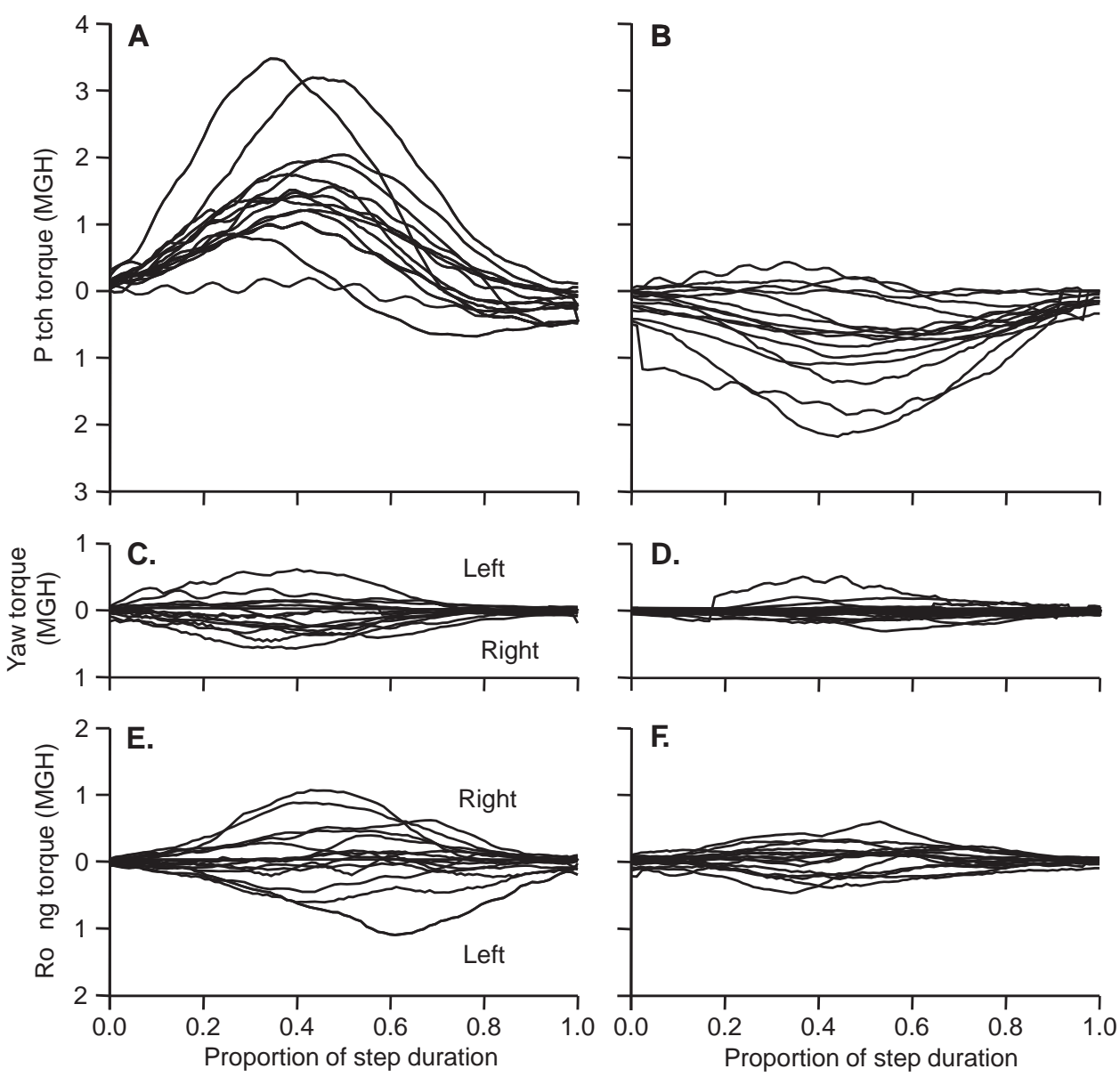

Fig. 8. Torques around the center of mass during forelimb contacts (A, C, and E) and hindlimb contacts (B, D, and F).

significant differences in timing between limb pairs. Hindlimbs usually made contact as soon as the forelimbs lifted off, making the torque generation more or less continuous. Hindlimb pitch torque was almost always entirely negative (Fig. 8), and the peak occurred at about mid-step ( $56 \pm 16 \%$ ). Yaw and rolling torques usually peaked close to the middle of the step, but the variation (as measured by standard deviation) was considerably higher than for pitch torques.

\section{Discussion}

Our first hypothesis that the angular impulses around each axis would sum to zero is not supported by our data (Table 2 and Fig. 7). To make this assessment, we compared the mean net torques with the mean positive and negative torques, using only the trials where both fore-and hindlimbs contacted the force pole. The pitch torques are fairly close to balanced, although it was apparent that the angular impulses were nearly always greater in the positive direction as compared with the negative direction. One plausible explanation for this finding is that the center of mass has shifted to a more anterior position in the body during the time of hindlimb contact. During forelimb contact, the abdominal contents and more massive hindlimbs shift anteriorly within the body (Carrier, 1987; forelimb and hindlimb mass data are unpublished, resulting from a single sacrificed chipmunk). If, during hindlimb contact, the center of mass is more anterior than our measurements could determine, the moment arm will be longer between the substrate reaction force and the center of mass. The longer moment arm would make the positive and negative pitch torques more balanced. Likewise, during the latter portion of the forelimb contact (when at least some of the abdominal and hindlimb mass has shifted anteriorly), the more anterior position of the center of mass relative to the hand position will decrease the positive pitch torque or increase the negative pitch torque.

During in-phase (asymmetrical) gaits, which include the bounding gait used by the chipmunk as well as galloping and halfbounding, the posterior 5-7 vertebrae and the sacrum flex and extend to a considerable extent (Schilling and Hackert, 2006). It

Table 4

Mean ( \pm standard deviation) timing of peak torques relative to step duration for forelimbs $(N=14)$ and hindlimbs $(N=15)$.

\begin{tabular}{|c|c|c|c|c|}
\hline & \multicolumn{2}{|l|}{ Positive } & \multicolumn{2}{|l|}{ Negative } \\
\hline & Forelimbs & Hindlimbs & Forelimbs & Hindlimbs \\
\hline Pitch (\% step duration) & $40 \pm 6$ & $52 \pm 43$ & $92 \pm 7$ & $57 \pm 16^{*}$ \\
\hline Yaw (\% step duration) & $55 \pm 34$ & $54 \pm 28$ & $49 \pm 29$ & $45 \pm 29$ \\
\hline Roll (\% step duration) & $49 \pm 27$ & $46 \pm 23$ & $47 \pm 36$ & $38 \pm 32$ \\
\hline
\end{tabular}

${ }^{*}$ Forelimbs and hindlimbs significantly different, $P \leq 0.001$. 


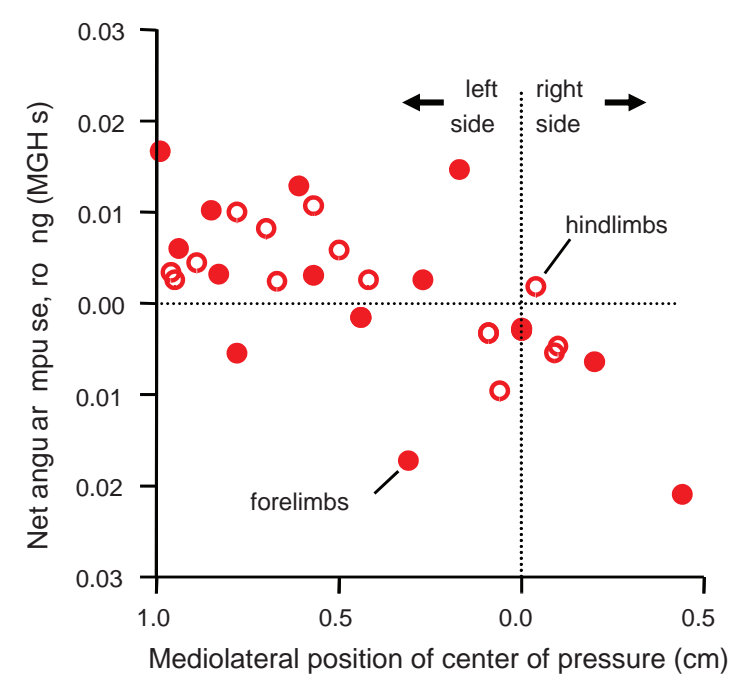

Fig. 9. Net rolling angular impulse versus mediolateral position of the center of pressure on the branch trackway. Forelimb contacts are represented by filled circles, hindlimb contacts by open circles.

is possible that the positive and negative pitch torques contribute to the flexion and extension of the spine and body as well as to the rotation of the body around the mediolateral axis passing through the center of mass. We believe, however, that pitch torques contribute mostly to the rotation of the whole body in the sagittal plane because Schilling and Carrier (2010) found that the epaxial muscles of dogs are active during galloping in such a way so as to cause extension. Therefore, the substrate reaction force causes the whole body to rotate in the sagittal plane, while the epaxial muscles cause the within-body movement in the sagittal plane. Furthermore, Reilly et al. (2010) demonstrated that hypaxial muscles are also active during locomotion, although the mammals they studied were trotting marsupials rather than bounding or galloping eutherians. Thus we agree with the conclusions of Bertram and Gutmann (2008): the substrate reaction forces function during the bound or gallop in such a way as to re-orient the body for the next aerial phase. Flexion and extension of the torso are accomplished via epaxial and hypaxial musculature, and the substrate reaction torques contribute a little or not at all.

When we explored the relationship between yaw and rolling angular impulses separately for forelimbs and hindlimbs, we found a rather high positive correlation in the forelimbs. For example, when rolling angular impulse is strongly positive (causing the animal's body to roll to the right and possibly topple), a similarly strong positive yaw angular impulse is exerted (causing the animal's body

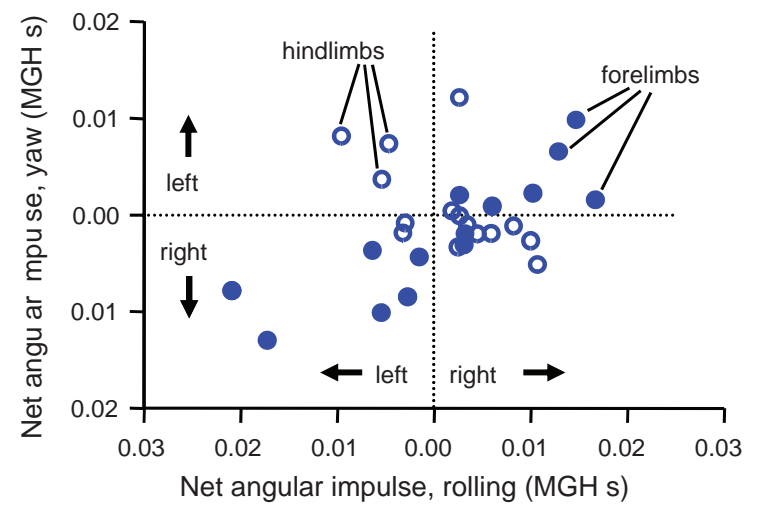

Fig. 10. Net yaw angular impulse versus net rolling angular impulse. Forelimb contacts are represented by filled circles, hindlimb contacts by open circles. to turn to a heading to the left). We found the opposite pattern in the hindlimb yaw and rolling angular impulses, although the correlation was much less strong. It is possible that when the chipmunks begin to topple to one side of the branch trackway, they exert a yaw torque in the opposite direction to avoid a fall. However, another intriguing possibility exists. The strong pitch angular momenta cause a good deal of rotation in the animal's body around a mediolateral axis. It is possible that when a tendency to topple occurs (because of an excessive rolling angular momentum), the pitch angular momentum acts like a gyroscope and produces a yaw torque that counters the rolling. A similar effect occurs in a moving bicycle, where the rotation of the wheels (analogous to the pitch movement in the chipmunks) prevents toppling to the side (analogous to excessive rolling in the chipmunks) by generating a yaw torque to oppose the topple (French, 1971).

Net rolling angular impulses were usually unbalanced within a single stride, and net yaw angular impulses were also unbalanced but to a lesser extent. Even if we allow for some inaccuracy in measuring the center of mass position, the positive and negative yaw and rolling torques rarely sum to zero within a stride. Each set of torque data was gathered within individual strides, and we suspect that if we gathered yaw and roll torque data over the course of two or three consecutive strides, the net angular impulses (positive angular impulse + negative angular impulse) would be much closer to zero. The possibility that each stride at steady speed is not more or less exactly like the previous and successive stride is an important result because throughout the field of locomotor biomechanics, usually a full set of variables (e.g., footfall pattern, substrate reaction force, limb kinematics) is gathered only from a single stride for each trial or case. Forner-Cordero et al. (2006) suggest that the stride is "quasi-periodic", and our data show the same. Belli et al. (1995) even go so far as to recommend that 32-64 consecutive strides should be analyzed to obtain accurate results on biomechanic variables, although their study analyzed locomotion on a treadmill. We predict that over the course of two or three strides, the net yaw and rolling angular impulses are much closer to zero. We think the chipmunks remain dynamically stable by fluctuating between positive and negative yawing and rolling, but always keeping fluctuations within a range. Such a way of regulating a biological activity is nothing new - for example, the parasympathetic and sympathetic nervous systems keep the heart rate within a range so that the heart rate at any moment is never far from the mean heart rate.

Among Siberian chipmunks running on an arboreal trackway, pitch angular impulses were greatest in magnitude, followed by rolling and then yaw angular impulses. This pattern also holds true for walking humans (Herr and Popovic, 2008), trotting goats (Biewener et al., 2009), and galloping dogs and goats (Biewener et al., 2009), but not for cockroaches using an alternating-tripod gait (Ting et al., 1994). When comparing relative magnitudes of pitch, roll, and yaw angular impulses between humans and chipmunks, it is not surprising that chipmunk pitch is far greater because they were bounding. Biewener et al. (2009) also found that pitch torque was considerably higher during galloping as opposed to trotting. We think it is likely that pitch angular impulses will be much smaller if the chipmunks trot or walk, and that yaw angular impulse would be relatively greater (Ting et al., 1994). Rolling angular impulses would have been lower if the chipmunks were not biased toward running on the left side of the branch. We assume this bias arose because the chipmunks were attempting to stay away from the light of the strobes, the human experimenters, or other factors. We did not attempt to remove the left-side-biased data because we assume that animals that regularly travel on branches will sometimes run on the left or right side of a narrow branch to avoid being seen by predators or to keep out of the wind. 
Chipmunks are proficient at running on arboreal supports, but tree squirrels are obviously much more so. We intend to compare angular impulses (among other biomechanic variables) between the scansorial chipmunks and the arboreal specialist squirrels of similar size. We anticipate that among tree squirrels, all net angular impulses within a stride will be much closer to zero as compared with chipmunks. Tree squirrels have presumably evolved motor patterns that permit more refined dynamic stability on narrow arboreal supports.

\section{Acknowledgements}

We thank Marissa Allen, Andrew Leith, Katie Rathers, Kelley Sufka, and Magdalena Wilczek for their help collecting and processing data, and Beth Judy for animal care. John Bertram provided key insights into the design of the force transducers and the concepts explored in this paper. Anonymous reviewers provided helpful comments on the manuscript. We are grateful to the College of Sciences and Health Professions, the Engaged Learning Summer Undergraduate Research program, and the University Research Council Faculty Research Development Program at Cleveland State University for funding.

\section{References}

Belli, A., Lacour, J.-R., Komi, P.V., Candau, R., Denis, C., 1995. Mechanical step variability during treadmill running. Eur. J. Appl. Physiol. Occup. Physiol. 70, 510-517.

Bertram, J.E.A., Gutmann, A., 2008. Motions of the running horse and cheetah revisited: fundamental mechanics of the transverse and rotary gallop. J. R. Soc. Interface 6, 549-559.

Biewener, A.A., Ros, I., Lee, D.V., Antonnen, J., Higgins, T., 2009. Ground reaction force and center of mass dynamics of goats and dogs during trotting and galloping. Int. Comp. Biol. 49, E15.

Carrier, D.R., 1987. The evolution of locomotor stamina in tetrapods: circumventing a mechanical constraint. Paleobiology 13, 326-341.

Cartmill, M. 1985. Climbing. In: Hildebrand, M., Bramble, D.M., Liem, K.F., Wake, D.B. (Eds.), Functional Vertebrate Morphology. Belknap Press of Harvard University Press, Cambridge, MA, pp. 73-88.

Elliot, L., 1978. Social behavior and foraging ecology in the Eastern Chipmunk(Tamias striatus) in the Adirondack Mountains. Smithson. Contrib. Zool. 265, 1-107.
Forner-Cordero, A., Koopman, H.J.F.M., van der Helm, F.C.T., 2006. Describing gait as a sequence of states. J. Biomech. 39, 948-957.

French, A.P., 1971. Newtonian Mechanics. The M.I.T. Introductory Physics Series W.W. Norton, New York

Herr, H., Popovic, M., 2008. Angular momentum in human walking. J. Exp. Biol. 211, 467-481.

Hildebrand, M., 1976. Analysis of tetrapod gaits: general considerations and symmetrical gaits. In: Miterman, R., Griller, S., Stein, P.S.G., Stuart, D.G. (Eds.), Neural Control of Locomotion. Plenum Press, New York, pp. 203-236.

Kawamichi, M., 1989. Nest structure dynamics and seasonal use of nests by Siberian chipmunks (Eutamias sibiricus). J. Mammal. 70, 44-57.

Lammers, A.R., Biknevicius, A.R., 2004. The biodynamics of arboreal locomotion: the effects of substrate diameter on locomotor kinetics in the gray short-tailed opossum (Monodelphis domestica). J. Exp. Biol. 207, 4325-4336.

Lammers, A.R., Earls, K.D., Biknevicius, A.R., 2006. Locomotor kinetics and kinematics on inclines and declines in the gray short-tailed opossum Monodelphis domestica. J. Exp. Biol. 209, 4154-4166.

Nauwelaerts, S., Kaiser, L., Malinowski, R., Clayton, H.M., 2009. Effects of trunk deformation on trunk center of mass mechanical energy estimates in the moving horse, Equus caballus. J. Biomech. 42, 308-311.

Özkaya, N., Nordin, M., 1999. Fundamentals of Biomechanics, 2nd ed. Springer, New York.

Piaggio, A.J., Spicer, G.S., 2001. Molecular phylogeny of the chipmunks inferred from mitochondrial cytochrome $b$ and cytochrome oxidase II gene sequences. Mol Phylog. Evol. 20, 335-350.

Preuschoft, H., 2002. What does "arboreal locomotion" mean exactly and what are the relationships between "climbing", environment and morphology? Z. Morphol. Anthrop. 83, 171-188.

Reilly, S.M., McElroy, E.J., White, T.D., Biknevicius, A.R., Bennett, M.B., 2010. Abdominal muscle and epipubic bone function during locomotion in Australian possums: insights to basal mammalian conditions and eutherian-like tendencies in Trichosurus. J. Morphol. 271, 438-450.

Schilling, N., Carrier, D.R., 2010. Function of the epaxial muscles in walking, trotting and galloping dogs: implications for the evolution of epaxial muscle function in tetrapods. J. Exp. Biol. 213, 1490-1502.

Schilling, N., Hackert, R., 2006. Sagittal spine movements of small therian mammals during asymmetrical gaits. J. Exp. Biol. 209, 3925-3939.

Schmitt, D., Lemelin, P., 2002. Origins of primate locomotion: gait mechanics of the woolly opossum. Am. J. Phys. Anthrop. 118, 231-238.

Stevens, N.J., 2003. The influence of substrate size, orientation and compliance upon prosimian arboreal quadrupedalism. Ph.D. Thesis. State University of New York at Stony Brook.

Ting, L.H., Blickhan, R., Full, R.J., 1994. Dynamic and static stability in hexapedal runners. J. Exp. Biol. 197, 251-269.

Youlatos, D., Michael, D.M., Tokalaki, K., 2008. Positional behavior of Siberian chipmunks (Tamias sibiricus). J. Ethol. 26, 51-60.

Post-print standardized by MSL Academic Endeavors, the imprint of the Michael Schwartz Library at Cleveland State University, 2017. 